\title{
Determination of temperature dependences of Young's modulus and internal friction of fuel cladding by resonance method
}

\author{
$A$ A Moshev, $S P$ Martynenko, $S S$ Martynenko, $E M$ Kudryavtsev, $A N$ Ableyev, $A N$ \\ Tokarev, $R A$ Panasenko, I I Rodko, $A$ I Maksimkin*, $Y O$ Zhukovskii, and $E G$ \\ Belendryasova \\ National Research Nuclear University MEPhI (Moscow Engineering Physics Institute), 115409 \\ Moscow, Russia
}

\begin{abstract}
We study elastic characteristics and internal friction of fuel claddings to improve computer codes for VVER-1000 fuel rods. We analytically described elastic characteristics of cladding material and obtained coefficient of the form of the first longitudinal frequency numerically. We described new measuring module for automatic acquisition data. We've established temperature dependences of Young's modulus and internal friction via high-temperature facility and developed electronic module and noted maximum of these characteristics at the temperature $1160 \mathrm{~K}$. It can be explained by the destruction of the texture in the material of claddings.
\end{abstract}

\section{Introduction}

The development of new types of nuclear fuel involves the study of their physical and mechanical properties [1]. One of the most important parameters required for fuel rod codes is the temperature dependence of the elastic characteristics (Young's modulus, shear modulus, Poisson's ratio) of the fuel rod cladding material in the operating temperature range. This paper describes the resonance method [2, 3], ultrasonic facility and experimental results on the temperature dependences of Young's modulus and internal friction of fuel rod claddings from zirconium alloys. The measurements were carried out via special measuring module developed in NRNU MEPhI. A distinctive feature of the module is a uniform amplitude- frequency characteristic of the measuring channel in the frequency interval $20-200 \mathrm{kHz}$ with its high sensitivity and noise immunity, which allows measurements of elastic characteristics and internal friction of samples in a wide range of their $q$-factor. In this case, the sample is clamped between the pointed ends of the acoustic waveguides, one of which provides the transfer of vibrations from exciting piezoelectric transducer to the sample, and the second - from the sample to receiving transducer [4-7].

The facility allows to measure the temperature dependence of the characteristics of elasticity and internal friction of a wide class of products of different sizes, including composites, fuel cladding, powder metallurgy products, etc. up to their melting

\footnotetext{
* Corresponding author: aimaksimkin@mephi.ru
} 
temperatures, as well as to investigate the features of structural and phase transformations in them, the kinetics of sintering of powder metallurgy products, to carry out flaw detection of serial products, to assess the quality of composites, including the adhesion of components, etc.

Our paper is structured as follows: in the section 2 we give basic theory states of our measurements. Experimental facility is described in the section 3. The results of experiments are presented in the section 4. In conclusion 5 we summarize important achievements.

\section{Theoretical basis of measurements}

The principle of operation of the facility is based on the relationship of the resonance characteristics of the samples (resonance frequencies $f_{i}$ and $Q_{i}$, where $i$ is the number of the resonance peak) with their elastic and inelastic characteristics.

The elasticity characteristics of isotropic materials are: Young's modulus $E$, shear modulus $G$, bulk modulus of elasticity $B$, Poisson's ratio $v$. Of these four characteristics for isotropic materials, two are independent, since there are coupling equations:

$$
\begin{aligned}
& G=\frac{E}{2(1+v)} \\
& B=\frac{E}{3(1-2 v)}
\end{aligned}
$$

In General, the resonance spectrum of an arbitrary shape product is a function of several parameters. The frequencies $f_{i}$ of natural oscillations of any isotropic defect-free body with characteristic size $d$ can be represented as:

$$
f_{i}=P_{i}(d, v) C_{L}
$$

where $P_{i}(d, v)$ is the coefficient of the form of oscillations at the $i$-th frequency, which depends on the geometry of the product and its Poisson's ratio $v . C_{L}$ is compressional velocity in the product:

$$
C_{L}=\sqrt{\frac{E}{\rho}}
$$

where $E$ - Young's modulus, $\rho$ - density of material.

For products of the cylindrical form made of one material (for example, segments of claddings of fuel elements), the coefficient of the form can be represented as:

$$
P_{i}(d, v)=\frac{1}{2} h k_{i}
$$

where $h$ is the height of the sample; $k_{i}$ is the coefficient of the $i$-th mode of oscillations, depending on the ratio of height to the diameter of the segment.

For standard segments of claddings $30 \mathrm{~mm}$ long, the Young's modulus $E$ is:

$$
E=4 h^{2} f_{c o m}^{2} k_{c o m} \rho
$$

And shearmodulus:

$$
G=4 h^{2} f_{\text {tor }}^{2} \rho
$$

where $f_{\text {com }}$ - the resonant frequency of the first longitudinal form of vibrations of the segment, $k_{\text {com }}$ - the coefficient of the form of the first longitudinal frequency, close to one, $f_{\text {tor }}$ - the resonant frequency of the first torsional form of vibrations of the fuel element segment.

The internal friction is determined by the formula: 


$$
Q_{i}^{-1}=\frac{\Delta f_{i}^{0.7}}{f_{i}}
$$

where $\Delta f_{i}^{0.7}$ is the width of the resonance peak at the height of 0.7 of the maximum amplitude.

The coefficient $k_{\text {com }}$ is calculated using the finite element method (FEM) in ANSYS for different lengths of fuel cladding segments. The calculation results are shown in figure 1.

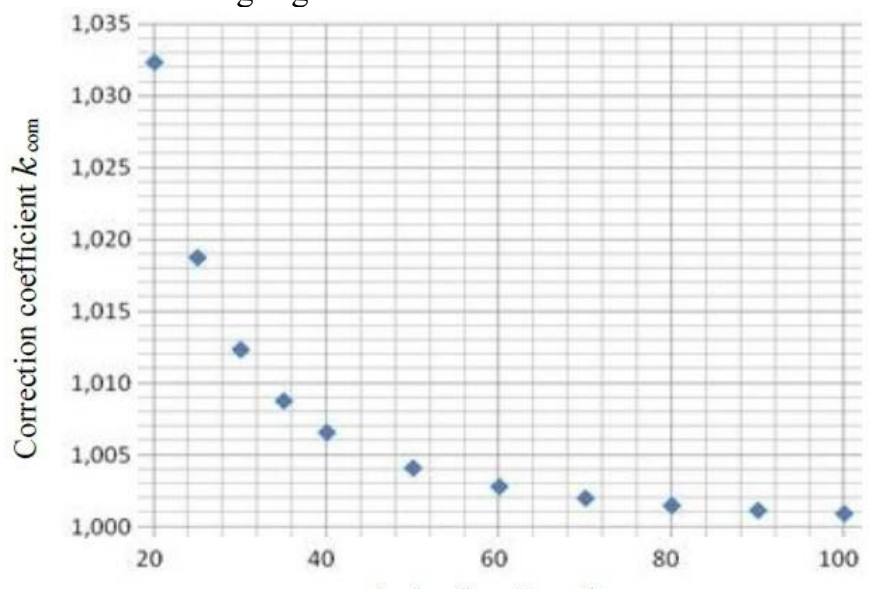

fuel rod cut length, $\mathrm{mm}$

Fig. 1. The dependence of the correction coefficient $k_{\text {com }}$ on the length of the fuel cladding segment.

\section{Experimental facility}

The block diagram of the experimental sample of a spectroscopic high-temperature facility for measuring the elastic characteristics and internal friction of the samples is shown in figure 2. The facility consists of two parts in the form of stainless steel pipes with a flange connection - a fixed 5 with an ultrasonic measuring module and a movable one with a heater 14. The segment of the cladding or the test sample 1 is clamped between two stainless steel acoustic waveguides, one of which 2 provides transfer of ultrasonic vibrations from exciting piezoelectric transducer to the segment of cladding, and the second 3 - from the cladding to receiving piezoelectric transducer. After that, the movable part is moved in the horizontal direction and is connected fixed via a flange gasket 4. Dampers 6 are used to optimize the amplitude-frequency characteristics of the measurement channel. At opposite ends of acoustic waveguides there are transducers - one 8 for exciting vibrations and 7 for receiving them. The electrical leads from the piezoelectric transducers and the chromiumaluminum thermocouple 10 pass through the rubber seal 9 . To generate sinusoidal signals in a given frequency range, register an informative signal and communicate with the control computer 13, the interface block 12 is used. The system of vacuuming and filling the facility with inert gas includes a nozzle 11, flexible hoses and a system 16, which provides an inert environment in the facility. The power supply of the heater 15 heats the nichrome heater 14 and is controlled by the computer 13 through the interface unit 12 . The main technical characteristics of the module are given in table 1.Measurements are carried out automatically using the PC. 


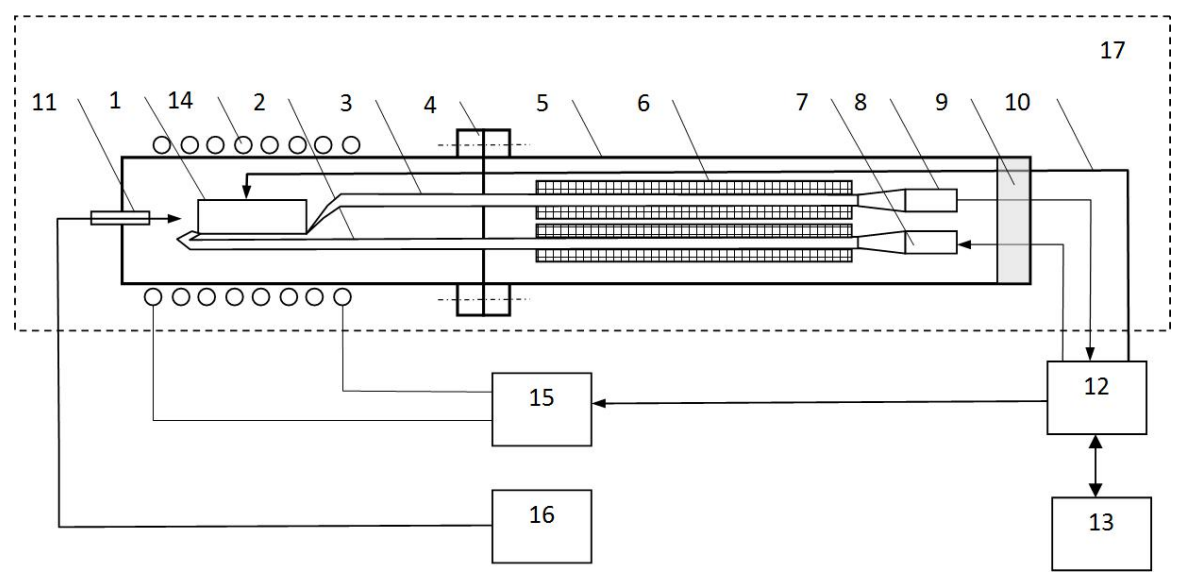

Fig. 2. Block diagram of the facility: 1 - short segment of a cladding; 2 - acoustic waveguide for the transfer of vibrations from the excitation piezoelectric transducer to the sample; 3 - acoustic waveguide for the transfer of vibrations from the sample to receiving piezoelectric transducer; $4-$ flange gasket; 5 - case; 6 - damper; 7 - exciting the piezoelectric transducer; 8 -receiving piezoelectric transducer; 9-flange gasket; 10 -chromel-aluminum thermocouple; 11 - nozzle of filling inert gas; 12 - the interface block; 13 - control computer; 14 - heater; 15- power supply to the heater; 16 - filling system with an inert gas; 17 - hot chamber.

Table 1. Main technical characteristics of the facility.

\begin{tabular}{|l|l|}
\hline Temperature measurement range & $300-1500 \mathrm{~K}$ \\
\hline Environment & Vacuum, inert \\
\hline Sample mounting method & point \\
\hline Measurement error of young's and shear modules, not more than \% & 1 \\
\hline The range of internal friction of samples & $10^{-4}-0,2$ \\
\hline Measurement error of internal friction, not more than \% & 5 \\
\hline The working range of frequencies & $20-200 \mathrm{kHz}$ \\
\hline Unevenness of the frequency response of the measuring path, dB & 6 \\
\hline Material of acoustic waveguides & Stainless steel \\
\hline The material of the transducer & PZT-19 \\
\hline
\end{tabular}

\section{Experimental results}

Via the developed facility, measurements of Young's modules, shear and internal friction of a series of fuel rod segments from zirconium alloys of promising compositions were carried out. In figures 3(a) and 3(b), the results obtained for the VVER standard cladding are presented in the form of temperature dependence graphs. It can be seen from the graphs that at the first heating of the samples (lower curve) at a temperature of $1160 \mathrm{~K}$ there is a characteristic change in Young's modulus and internal friction (the so-called peak of internal friction), indicating the phase transition in the samples from $\alpha$-Zr with a hexagonal lattice ( $a$ $=0.3231 \mathrm{~nm}, C=0.5146 \mathrm{~nm})$ to $\beta-\mathrm{Zr}$ with a cubic lattice $(a=0.361 \mathrm{~nm})$. In the subsequent cooling of samples from high temperatures, the young modulus increases in the temperature range of $300-1100 \mathrm{~K}$ by $10-20 \%$, which can be explained by the destruction of the existing texture in the cladding after the passage of phase transformations. 


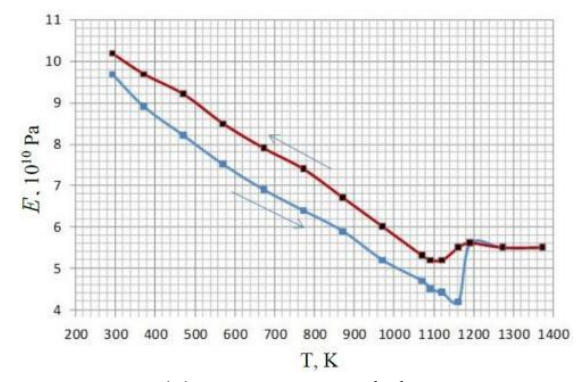

(a)Young's modulus

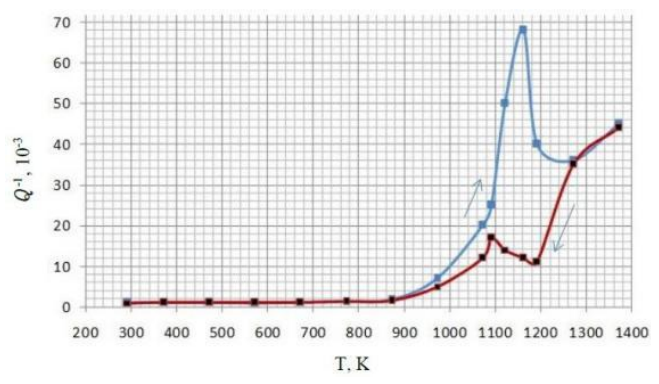

(b) Internal friction

Fig. 3. Temperature dependences of characteristics of standard cladding of VVER fuel rod on heating (blue curve) and cooling (red curve).

\section{Conclusion}

We developed ultrasonic module of high-temperature facility allows to measure the temperature dependence of elastic characteristics and internal friction of standard fuel rod claddings. Obtained characteristics behave non-monotonously in narrow temperature range $1100-1200 \mathrm{~K}$ and at the temperature 1160 there is a maximum - internal friction peak. Such phenomenon can be explained via the destruction of material texture.

We also found correction coefficient of the form the first longitudinal frequency via finite element method (see Figure 1).

The results obtained during the presented experimental work will become the fundamental for developing the system of integrated digital twins of fuel rods. The framework establishes the relationship between thermal-physical and neutron design codes with a change in the technical state of the fuel rods, determined by the methods presented.

\section{Acknowledgments}

The research was supported by the Ministry of science and higher education of the Russian Federation, the Agreement №14.578.21.0258, a unique identifier of the project RFMEFI57817X0258.

\section{References}

1. Reshetnikov F G, Bibilashvili Y K, Golovnin I S, Gorsky V V, Kazennov Y I, Menshikova T S, Nikulina A V and Romaneev V V 1995 Development, production and operation of fuel elements of power reactors vol 1 (Moscow: Energoatomizdat)

2. Baranov V M 1989 Acoustic measurements in nuclear energy (Moscow: Energoatomizdat)

3. Baranov V M, Karasevich A M, Kudryavtsev E M and et al 1999 Acoustic measurements in nuclear energy(Moscow: Energoatomizdat)

4. Maximkin A I, Kryukov I S, Ableev A N, Berestov A V and Rodko I I 2018 ICONE 2018 vol 3 (London, United Kingdom: American Society of Mechanical Engineers)

5. Martynenko S P, Martynenko S S, Ableev A N, Panasenko R A, Anufriev B F and Maksimkin A I 2016 ICCICCT 2015 (Kumaracoil, India: Institute of Electrical and Electronics Engineers) pp 668-671 [6]Martynenko S S, Ableev A N, Martynenko S P, Panasenko R A, Berestov A V and Rodko I I 2016 ICCICCT 2015 (Kumaracoil, 
India: Institute of Electrical and Electronics Engineers) pp 672-676 [7]Panasenko R A, Martynenko S P, Martynenko A S, Ableev A N, Kudryavtsev E M, Frolov E S and

6. Varyatchenko E P 2016 ICCICCT 2015 (Kumaracoil, India: Institute of Electrical and Electronics Engineers) pp 677-681 